\title{
A CLINICAL STUDY ON PALMAR DERMATOGLYPHICS IN PSORIASIS
}

\author{
Aswathy Umesh1, Priya Ashok2, Joan Felicita Samson ${ }^{3}$ \\ 1 Junior Resident, Department of Dermatology \& Venereology, Government Medical College, Trivandrum. \\ ${ }_{2}^{2}$ Assistant Professor, Department of Dermatology \& Venereology, Government Medical College, Trivandrum. \\ ${ }^{3}$ Professor, Department of Dermatology \& Venereology, Dr. Somervell Memorial CSI Medical College, Karakonam, Trivandrum.
}

\section{ABSTRACT}

\section{BACKGROUND}

There is dearth of data regarding the dermatoglyphic pattern in psoriasis patients of South India. The aim is to study the palmar dermatoglyphic pattern in psoriasis and to compare it with that of a comparison group.

\section{MATERIALS AND METHODS}

All consecutive psoriasis patients who attended the Department of Dermatology in Government Medical College, Thiruvananthapuram during a period of one and a half year were included in this descriptive comparative study.

\section{RESULTS}

The study population comprised of 103 patients. A similar number of normal population was considered as a comparison group. In fingertip patterns, loops were the predominant pattern followed by whorls and arches in psoriasis patients. In thumb of both hands, the number of whorls were high in psoriasis patients compared to comparison group. In index finger of both hands, the number of arches were high and whorls were low when compared to comparison group. A low whorl pattern in middle finger (right and left) and a high arch pattern in left index finger were observed in psoriasis patients. A low ulnar loop pattern in left ring finger, low whorl pattern in right ring finger and a high arch pattern in both ring fingers were observed in psoriasis patients. A high number of arch patterns were observed in both little fingers. An increase in true patterns was observed in right interdigital space I. The mean total finger ridge count was high in psoriasis patients.

\section{CONCLUSION}

Dermatoglyphics can be used as a cost-effective tool to predict the occurrence of psoriasis in a person. Dermatoglyphic analysis can be included in examination of persons who are susceptible to psoriasis (in case of family history of psoriasis) and in psoriasis patients, due to its advantages such as easy method of taking palm prints, fast results and cost-effectiveness.

\section{KEYWORDS}

Dermatoglyphics, Psoriasis, South India.

HOW TO CITE THIS ARTICLE: Umesh A, Ashok P, Samson JF. A clinical study on palmar dermatoglyphics in psoriasis. J. Evolution Med. Dent. Sci. 2017;6(60):4430-4434, DOI: 10.14260/Jemds/2017/957

\section{BACKGROUND}

Dermatoglyphics as defined by Cummins and Midlo (1926) is the scientific study of epidermal ridge configuration and ridges themselves. 'Derma' means skin, 'glyphics' means carvings, a word coined by the anatomist Harold Cummins of Tulane University. ${ }^{1}$ Wide spread interest developed in the last few decades with discovery of unusual ridge patterns in patients with chromosomal aberration and other medical diseases.

Dermatoglyphic patterns are genetically determined and are influenced by insults during early foetal life. Although once formed, the dermal configurations are resistant to environmental effects, they do reflect growth disturbances that occur before and during their development. ${ }^{2}$

Financial or Other, Competing Interest: None.

Submission 19-06-2017, Peer Review 13-07-2017,

Acceptance 21-07-2017, Published 27-07-2017.

Corresponding Author:

Dr. Priya Ashok,

Assistant Professor,

Department of Dermatology \& Venereology,

Government Medical College,

Trivandrum.

E-mail: priyaashok242@gmail.com

DOI: $10.14260 /$ jemds $/ 2017 / 957$

\section{(c) $($ () $(-)$}

A dermatoglyphic approach has been useful as a screening method for identifying patients who are likely to have chromosomal aberrations. It is a simple, inexpensive and atraumatic procedure and the taking of a good print makes a permanent and complete record. It can be easily included in the physical examination as a bedside procedure when a genetic disorder is suspected.

Dermatoglyphic disturbances in chromosomal aberrations such as Down syndrome, Trisomy 13, and Trisomy 18 are particularly striking and can be used to strengthen a diagnostic impression. Single gene mutations causing malformations of hands and feet such as syndactyly, polydactyly and brachydactyly are associated with dermatoglyphic features significantly varying from normal. There are in addition several disorders, both genetic and those not clearly genetically determined which although not proved to have caused any limb defects or pattern distortions may cause elevations of frequency distributions of certain characters or affect mean values. Those in this category range from congenital heart disease, spina bifida, diabetes mellitus, schizophrenia and leukaemia to dermatological entities such as psoriasis, vitiligo, and alopecia areata, Darier disease, discoid lupus erythematosus, ichthyosis, pemphigus and leprosy.

Psoriasis is a common papulosquamous disorder of multifactorial aetiology and hereditary tendency. 
Familial aggregation and concordance rate among mono zygotic twins emphasise the role of heredity in pathogenesis. Dermatoglyphics can be used to strengthen a diagnostic impression or to select persons for additional diagnostic studies. Nowadays, in biology, anthropology, genetics and medicine, dermatoglyphics serves as a tool to describe, compare, and contrast, and at times predict occurrences and risks for biomedical events.

This study was undertaken to study the dermatoglyphics in psoriasis and to compare it with that of normal population, so that it can be considered as an effective method for predicting the occurrence of psoriasis

\section{MATERIALS AND METHODS}

After obtaining clearance from the ethics committee patients with psoriasis who attended the Department of Dermatology, Government Medical College, Thiruvananthapuram for a period of one and a half years were evaluated. Patients with definite clinical diagnosis of psoriasis were taken as cases. This is a descriptive comparative study. Patients with age < 18 years, palmoplantar psoriasis, psoriasis with palmar involvement, pustular psoriasis, exfoliative dermatitis secondary to psoriasis, psoriatic erythroderma, and patients not willing for study were excluded. 103 patients with psoriasis were included in the study. Comparison group of 103 people without psoriasis were included in the study and comparison of dermatoglyphics between psoriasis patients and comparison group was done. (The sample size was calculated based on previous studies.)

Data collection was done using Interview method. An informed written consent was taken. Data including name, age, gender, address, occupation, educational status, income, OP number, phone number were recorded. Presenting complaints including that of skin, joint, mucosa, nail and hair were noted. Duration of illness, associated systemic diseases, history of addiction, drug intake were noted. Detailed general examination was done. Detailed dermatological examination and systemic examination were done. Investigations including blood routine examination, urine routine examination, liver function test, renal function test, serum calcium, serum uric acid and serum cholesterol were done in all cases. Collection of palm prints were done using fingerprint ink pads.

The comparison of fingertip pattern (Figure 1), interdigital pattern total finger ridge count, a-d ridge count, at dangle (figures 2\&3) in both groups were done. Qualitative variables were analysed using proportions. Quantitative variables were analysed using mean and standard deviation. Chi square test was applied to note if there is any statistical significance between the psoriasis patients and the comparison group and a ' $\mathrm{p}$ ' value less than 0.05 was considered statistically significant.

\section{RESULTS}

The predominant age group was 41-60 years which accounted for $68 \%$ of the cases. Majority of cases had a duration of illness of 1-5 yrs. Males outnumbered females with a male: female ratio of 1.39:1. Majority of cases were skilled manual labourers followed by unemployed persons. Majority of patients were married. Majority of patients had presenting complaint involving skin and nail (30.1\%) followed by patients with skin complaints alone (27.2\%).
$15.4 \%$ cases had joint involvement. $10 \%$ patients had involvement of joints. Among the 103 cases in this study, 53 cases had co morbidities, of which the most common was diabetes mellitus. Hypertension, dyslipidaemia, coronary artery disease were also associated. Out of the 53 patients with comorbidities, 32 patients (accounting for 60\%) had more than one comorbidity. A combination of diabetes mellitus, hypertension and dyslipidaemia was present in 2 cases.

History of addictions were present in 52 patients of which a majority had a habit of both smoking and alcoholism. The severity of psoriasis (as assessed by PASI score) was found to be high in patients with the habit of both smoking and alcoholism, when compared to patients with either smoking or alcoholism or pan chewing alone. The finding was statistically significant $(\mathrm{p}=0.04)$.

History of intake of beta blockers, NSAIDs, calcium channel blockers, ACE inhibitors, angiotensin receptor blockers were given by 16 patients. It was observed that majority of patients had a generalised involvement. The commonest mucosal change noted was fissured tongue followed by geographic tongue. Severity of psoriasis was more in patients with geographic tongue rather than those with fissured tongue. This finding was statistically significant $(\mathrm{p}=0.022)$.

Majority of patients (54.4\%) in the study had a PASI score between 1 to $18.80 .6 \%$ of patients had a PASI score $<37$. Nail involvement was observed in $56.3 \%$ cases. Cases with nail involvement had higher PASI score. But the finding was not statistically significant.

The prevalence of psoriatic arthropathy was 15.5\%. There were no significant differences in PASI score in comparison to joint involvement. $75 \%$ of the patients with psoriatic arthropathy had nail involvement also. Hypocalcaemia was observed in $28.2 \%$ cases. The severity of psoriasis was found to be higher in cases with hypocalcaemia. But the finding was not statistically significant.

\section{Dermatoglyphic Patterns}

The predominant pattern on thumb was ulnar loop. The number of whorls was high in psoriasis patients, whereas ulnar loops were low. The frequency of whorls was less in cases. The number of arches were low among psoriasis patients. In the index finger, arches were high and the number of whorls were low in psoriasis patients. This finding was statistically significant $(\mathrm{p}=0.004,0.009)$. There was a statistically significant difference in decreased whorl pattern in middle finger (right and left) $(p=0.016,0.017)$ and increased arches pattern (left) $(\mathrm{p}=0.019)$ in contrast to the comparison group.

Statistically significant difference was noted in patterns seen in left ring finger (a decrease in ulnar loop) ( $p=0.012)$, right ring finger (a decrease in whorl) $(p=0.003)$ and an increased arch pattern in both fingers (right and left) $(p=0.002,0.012)$. In the little finger, the number of arches were high and ulnar loops were low in psoriasis patients. These findings were statistically not significant.

Loops were the predominant pattern followed by whorls and arches in both right and left hand of psoriasis patients. It was observed that loops and arches were high and whorls and composite pattern low in male psoriasis patients. In 
females, loops were the predominant pattern. Increase in loops and decrease in whorls were statistically significant.

There was statistically significant difference in vestiges pattern in right interdigital space I in psoriasis patients than in comparison group $(\mathrm{p}=0.013)$.

The predominant pattern in interdigital space II was open space (Figure 3). But the differences between open spaces and true patterns were not statistically significant.

The predominant pattern in interdigital space III was open space followed by loops.

The predominant pattern in interdigital space IV (figure 4) was open space followed by loop.
The predominant pattern in hypothenar space was open space followed by loop.

There was a statistically significant increase in true patterns (loop/whorl/arch) in right interdigital space I.

Mean total finger ridge count (MTFRC) was high in psoriasis patients, but the result was not statistically significant.

There was no statistically significant difference in a-d ridge count in psoriasis patients and comparison group.

There was no statistically significant difference in atd angle in psoriasis patients and comparison group.

\begin{tabular}{|c|c|c|c|c|c|}
\hline \multirow{2}{*}{ Right } & \multicolumn{2}{|c|}{ Case } & \multicolumn{2}{|c|}{ Comparison Group } & \multirow{2}{*}{$P$ value } \\
\hline & True Patterns & Nil & True Patterns & Nil & \\
\hline Space I & 6 & 97 & 0 & 103 & 0.013 \\
\hline Space II & 8 & 95 & 7 & 103 & 0.789 \\
\hline Space III & 44 & 59 & 36 & 67 & 0.253 \\
\hline Space IV & 39 & 64 & 33 & 70 & 0.381 \\
\hline Hypothenar & 24 & 79 & 21 & 82 & 0.613 \\
\hline Left & True Patterns & Nil & True Patterns & Nil & \\
\hline Space I & 7 & 96 & 2 & 101 & 0.088 \\
\hline Space II & 7 & 96 & 3 & 100 & 0.195 \\
\hline Space III & 46 & 57 & 45 & 58 & 0.888 \\
\hline Space IV & 44 & 59 & 34 & 69 & 0.151 \\
\hline Hypothenar & 17 & 86 & 17 & 86 & 1.000 \\
\hline \multicolumn{6}{|c|}{ Table 1} \\
\hline
\end{tabular}

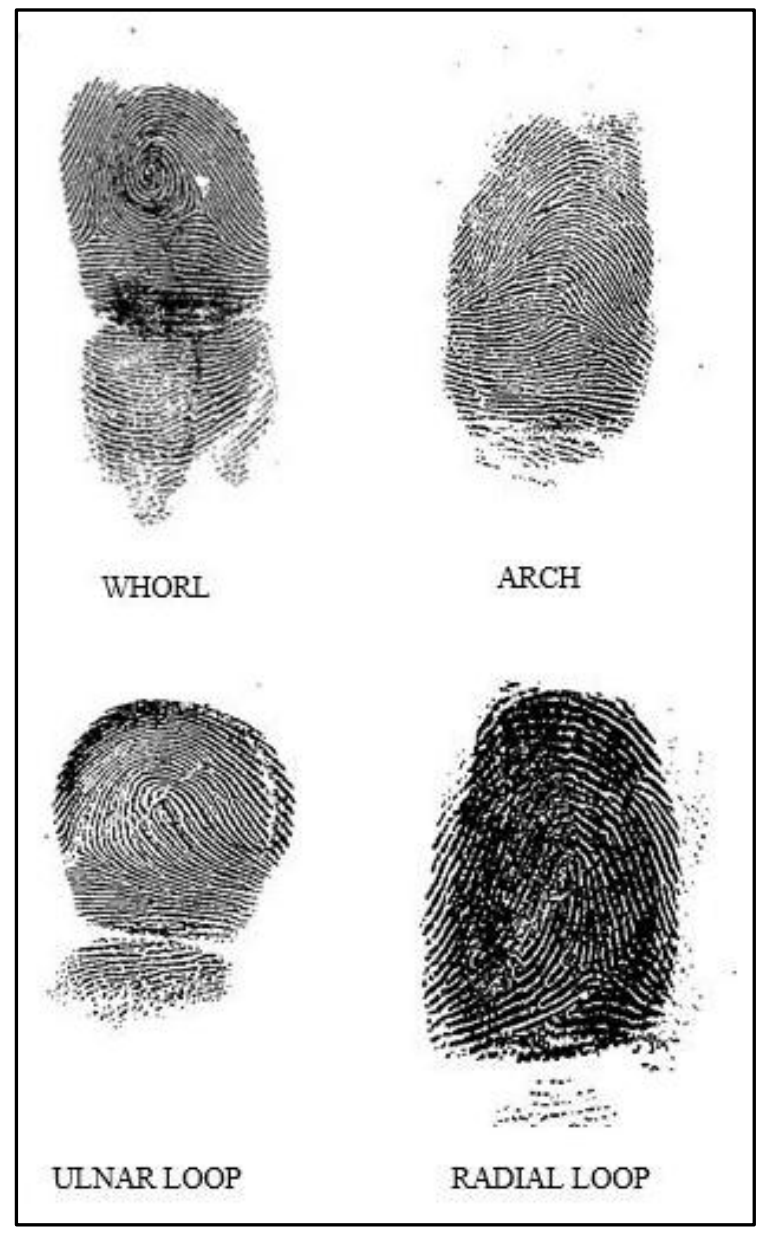

Figure 1

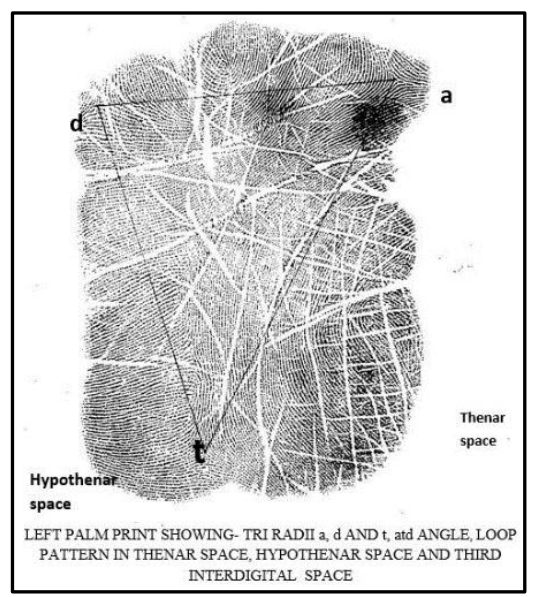

Figure 2

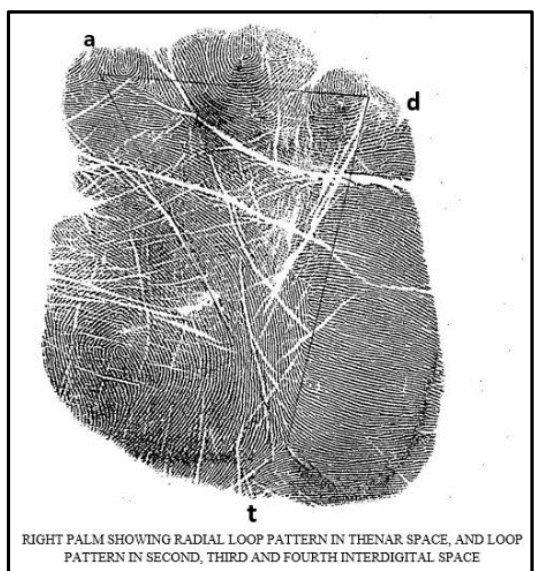

Figure 3 


\section{DISCUSSION}

Psoriasis has a bimodal peak regarding age of onset, first at 16-22 years and later at 57-60 years and there are two types namely type I and type II; according to age of onset before or after 40 years of life. ${ }^{3}$ So it can be inferred that majority of psoriasis patients in my study were type II.

In a previous study, a gender difference was found for both the PASI and the DLQI, with men often having high PASI scores and women more often having high DLQI scores. ${ }^{4}$ It may be explained by gender based differences of both innate and adapted immune responses in immunological diseases like psoriasis. ${ }^{5}$ Increase in severity of psoriasis in manual labourers may be due to an increased chance of trauma and subsequent koebnerisation in manual labourers.

It was observed that majority of patients had a generalised involvement followed by involvement of upper and lower limb. This is in contrast to previous studies where trunk was the most common site affected.6,7 These discordant findings may be due to the fact that this study was conducted in a tertiary care centre where more severe and generalised disease process are encountered. The frequency of geographic tongue increased with severity of psoriasis.

In the study, cases with nail involvement had higher PASI score. But the finding was not statistically significant. Nail psoriasis proves to have predictive value. The severity of psoriasis was found to be higher in cases with hypocalcaemia. But the finding was not statistically significant.

\section{Dermatoglyphic Patterns}

In fingertip patterns, loops were the predominant pattern followed by whorls and arches in psoriasis patients. In thumb of both hands, the number of whorls were high in psoriasis patients compared to comparison group. In index finger of both hands, the number of arches were high and whorls were low when compared to comparison group.

A low whorl pattern in middle finger (right and left) and a high arches pattern in left index finger were observed in psoriasis patients.

A low ulnar loop pattern in left ring finger, low whorl pattern in right ring finger and a high arch pattern in both ring fingers were observed in psoriasis patients.

A high number of arch patterns were observed in both little fingers.

An increase in true patterns was observed in right interdigital space I.

The mean total finger ridge count was high in psoriasis patients.

Analysis of ATD angle and a-d ridge count revealed no difference between psoriasis patients and comparison group. Even after an extensive search in databases, a similar study on comparison of a-d ridge count in psoriasis patients to a control group could not be accessed. It seems that this study is the first one to be undertaken to assess the parameter of a$\mathrm{d}$ ridge count in psoriasis patients.

All the other findings were concordant with few previous studies and discordant with other studies.8,9,10,11,12,13,14,15 This could be accounted by the racial differences in population under study in each of these studies. A similar study with the study population similar to the racial group in this study could not be found after an extensive data search. All the previous studies done in India were from north India where the racial groups undertaken were different from the
Dravidian race in South India evaluated in our study. Our study seems to be the first study done on the Dravidian race. To identify a person susceptible to psoriasis, an extensive study is warranted to establish a baseline data on the dermatoglyphics of the Dravidian population. Such a study can only provide an insight for practitioners in South India.

\section{Limitations}

Since the calculated sample size was too high and thereby not feasible to include in this limited period of study, we had to limit the sample size for convenience.

\section{CONCLUSION}

The observations from this study as well as from previous studies point to the fact that these findings can be used as tools to strengthen the diagnostic accuracy. The findings suggest that alterations in digital patterns may be a pleiotropic effect of the genes which produces the tissue changes of psoriasis.

Dermatoglyphic analysis can be included in examination of persons who are susceptible to psoriasis (in case of family history of psoriasis) and in psoriasis patients, due to its advantages such as easy method of taking palm prints, fast results and cost-effectiveness.

Although numerous dermatoglyphic studies have been reported from time to time with reference to various clinical and non-clinical conditions, there is dearth of information related to study of dermatoglyphic patterns with reference to psoriasis. So it warrants further extensive study to deduce correlations between the two in the Indian subcontinent as well as globally.

\section{REFERENCES}

[1] Verbov J. Clinical significance and genetics of epidermal ridges-a review of dermatoglyphics. J Invest Dermatol 1970;54(4):261-71.

[2] Miller JR. Dermatoglyphics. J Invest Dermatol 1973;60(6):435-42.

[3] Griffiths CEM, Barker JNWN. Psoriasis. In: Burns T, Breathnach S, Cox N, et al. (eds). Rook's textbook of dermatology. $8^{\text {th }}$ edn. Vol 2. West Sussex: Blackwell Publishing Ltd. 2010:1-60.

[4] Lesuis N, Befrits R, Nyberg F, et al. Gender and the treatment of immune-mediated chronic inflammatory diseases: rheumatoid arthritis, inflammatory bowel disease and psoriasis: an observational study. BMC Med 2012;10:82.

[5] Hagg D, Eriksson M, Sundstrom A, et al. The higher proportion of men with psoriasis treated with biologics may be explained by more severe disease in men. PLoS ONE 2013;8(5):e63619.

[6] Sinniah B, Devi SS, Prashant BS. Epidemiology of psoriasis in Malaysia: a hospital based study. Med J Malaysia 2010;65(2):112-4.

[7] Bedi TR. Clinical profile of psoriasis in North India. Indian J Dermatol Venereal Leprol 1995;61(4):202-5.

[8] Dehankar R, Bhakare U, Ksheerasagar DD. The study of palmar dermatoglyphics in psoriasis. Int J Pharm Bio Sci 2013;4(1):554-61.

[9] Gibbs RC, Warburton D. Studies of dermatoglyphics in psoriasis. J Invest Dermatol 1968;51:259-61. 
[10] Elizabeta C, Lazarova D, Trpkovska B, et al. Dermatoglyphics in patients with psoriasis. Acta Morphol 2013;10(1):15-8.

[11] Kumar P, Gupta A. Dermatoglyphic patterns in psoriasis, vitiligo and alopecia areata. Indian J Dermatol Venereol Leprol 2010;76(2):185-6.

[12] Verma KC, Joshi RK, Jain VK. Dermatoglyphics in psoriasis. Indian J Dermatol Venereol Leprol 1980;46(1):28-30.
[13] Nagar KS, Laha NN, Sethi NC. Palmar dermatoglyphics in psoriasis-a pilot study. Indian J Dermatol Venereol Leprol 1981;47(4):197-201.

[14] Kapur RT, Verma DR. Study of dermatoglyphics in dermatosis. Indian J Dermatol Venereol Leprol 1982;48(4):193-7.

[15] Sharma KN, Sarin RC, Prabhakar BR. Study of dermatoglyphics in dermatosis. Indian J Dermatol Venereol Leprol 1977;43(5):262-5. 